\title{
Pseudomelanomas After Conservative Management of Uveal Melanoma
}

\author{
W. R. LEE \\ Glasgow
}

\begin{abstract}
Summary
This report describes the macroscopic and microscopic features of 'pseudomelanomas' encountered in 48 globes containing malignant melanomas, which were initially treated either by local resection (45), by $\mathrm{Ru}^{106}$ plaque (2) therapy or by photocoagulation (1) therapy. Pigmented masses which simulated melanomas were formed by

(i) organising haemorrhage,

(ii) melanomacrophagic reactions to melanin released from necrotic tumours,

(iii) reactionary proliferations in the retinal pigment epithelium.
\end{abstract}

Fibroblastic reactions in response to photocoagulation or organising haemorrhage also produced pseudotumours.

The various disease entities which can lead to a false positive diagnosis of malignant melanoma of the uvea in untreated eyes (pseudomelanomas) are well documented., ${ }^{1,2,3}$ In summary, at the microscopic level, the following cells can accumulate or proliferate to form a pigmented mass: red cells, haemomacrophages, melanomacrophages and retinal pigmented epithelial cells. Paler tumours can be produced by choroidal exudates, metastatic tumours and metaplastic retinal pigment epithelial cells.

The application of new treatment modalities to malignant melanomas of the ciliary body and choroid has introduced modifications to the previously documented patterns of pathology. The purpose of this presentation is to describe some of the reactionary changes which have led to difficulty in macroscopic identification of residual tumour in enucleated eyes in which conservative management has been unsuccessful.

\section{Material and Methods}

Between 1969 and December 1986, 173 locally resected choroidal and ciliary body malignant melanomas were submitted for pathological examination. In 13 cases the globe was enucleated within one month of surgery and 32 eyes were enucleated within 1-3 years after the primary excision (for details of complications see reference 4). In addition, two globes had been treated by Ruthenium ${ }^{106}$ plaques, one by a $\mathrm{Co}^{60}$ plaque and one tumour was treated by photocoagulation only. In many of the local resection cases, recurrent tumour growth had been controlled subsequently for some time by photocoagulation and/or irradiation.

The enucleated eyes were fixed in glutaraldehyde (2 per cent) buffered with cacodylate. External examination of the globe with a dissecting microscope revealed scar tissue over the site of previous intervention and where present, extraocular spread of recurrent tumour. The globes were opened after the vortex veins were removed, in a plane which could best demonstrate the resection bed. In 42 of the globes initially treated by local resection, the

Correspondence to: Professor W. R. Lee, MD, FRCPath, Pathology Department, Western Infirmary, Glasgow G116NT.

Presented at the Annual Congress of the Ophthalmological Society of the United Kingdom April 1987. 
presence of residual or recurrent tumour was demonstrated.

Blocks were taken from the pupil-optic nerve axis and from any site in which there was evidence to suggest abnormal pigmentation or nodularity adjacent to, or distant from, the site of surgery or the other treatment modalities. The tissue blocks were processed for paraffin embedding and studied by serial section when necessary. Conventional stains used as a routine were: HE, PAS, Masson, Masson-Fontana, Melanin bleach, Prussian Blue, Holmes and Loyez. A detailed account of this series will be published at a later date.

\section{Results}

The pseudomelanomas which were noteworthy in this series were due to:

(a) unusual forms of organisation of subretinal haemorrhage;

(b) tumour necrosis and melanomacrophagic responses (seen as a response to irradiation and photocoagulation);

(c) fibroblastic proliferation derived from activated scleral fibroblasts (a response to surgery and photocoagulation);

(d) hypertrophy, hyperplasia and metaplasia in the retinal pigment epithelium (a response to surgery).

\section{(a) Organising haemorrhage}

In many specimens there was dark red or brown pigmentation of the chorioretinal tissue at the edge of the reaction (Fig. 1a). This was due to a proteinaceous exudation and accumulation of red cells and macrophages containing iron and other breakdown products of blood in the subretinal space. In some cases these macrophages were infiltrating the retina individually and in clusters (Fig. 1b) and the cytoplasm also contained melanin in addition to blood breakdown products.

In some cases of haemosiderosis, the retinal pigment epithelium stained positively for iron also. The source of the bleeding was neovascularisation at the edge of the surgical coloboma.
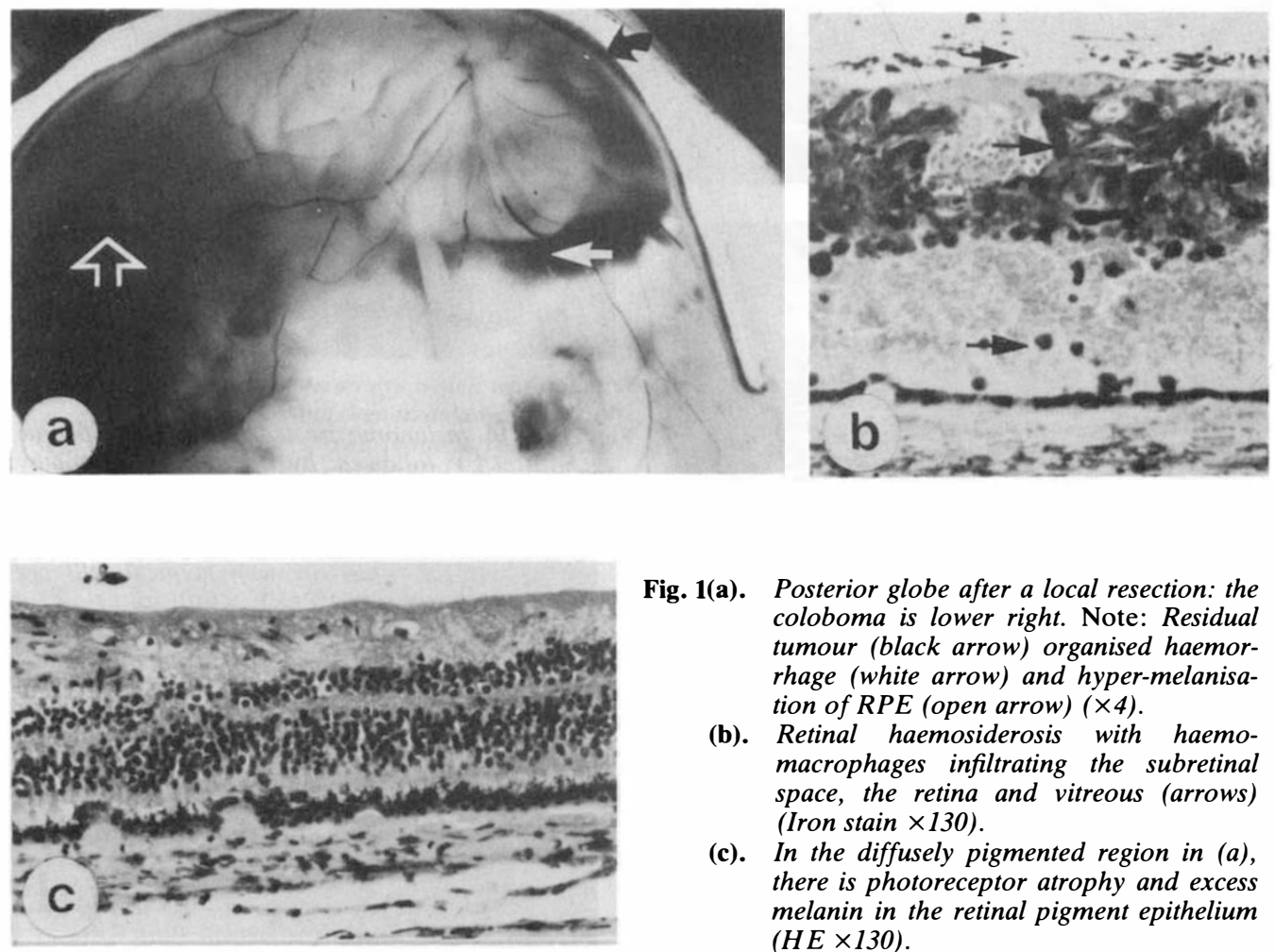

Fig. 1(a). Posterior globe after a local resection: the coloboma is lower right. Note: Residual tumour (black arrow) organised haemorrhage (white arrow) and hyper-melanisation of RPE (open arrow) (×4).

(b). Retinal haemosiderosis with haemomacrophages infiltrating the subretinal space, the retina and vitreous (arrows) (Iron stain $\times 130$ ).

(c). In the diffusely pigmented region in (a), there is photoreceptor atrophy and excess melanin in the retinal pigment epithelium $(H E \times 130)$. 
(b) Tumour necrosis and melanomacrophagic response

The most impressive example of melanomacrophagic activity in response to tumour cell necrosis was seen in a tumour which was treated by an $\mathrm{Ru}^{106}$ plaque (Fig. 2). To a lesser extent, a similar reaction was frequently noted in globes in which recurrences had been treated by photocoagulation. In these specimens the pseudomelanomas consisted of large numbers of
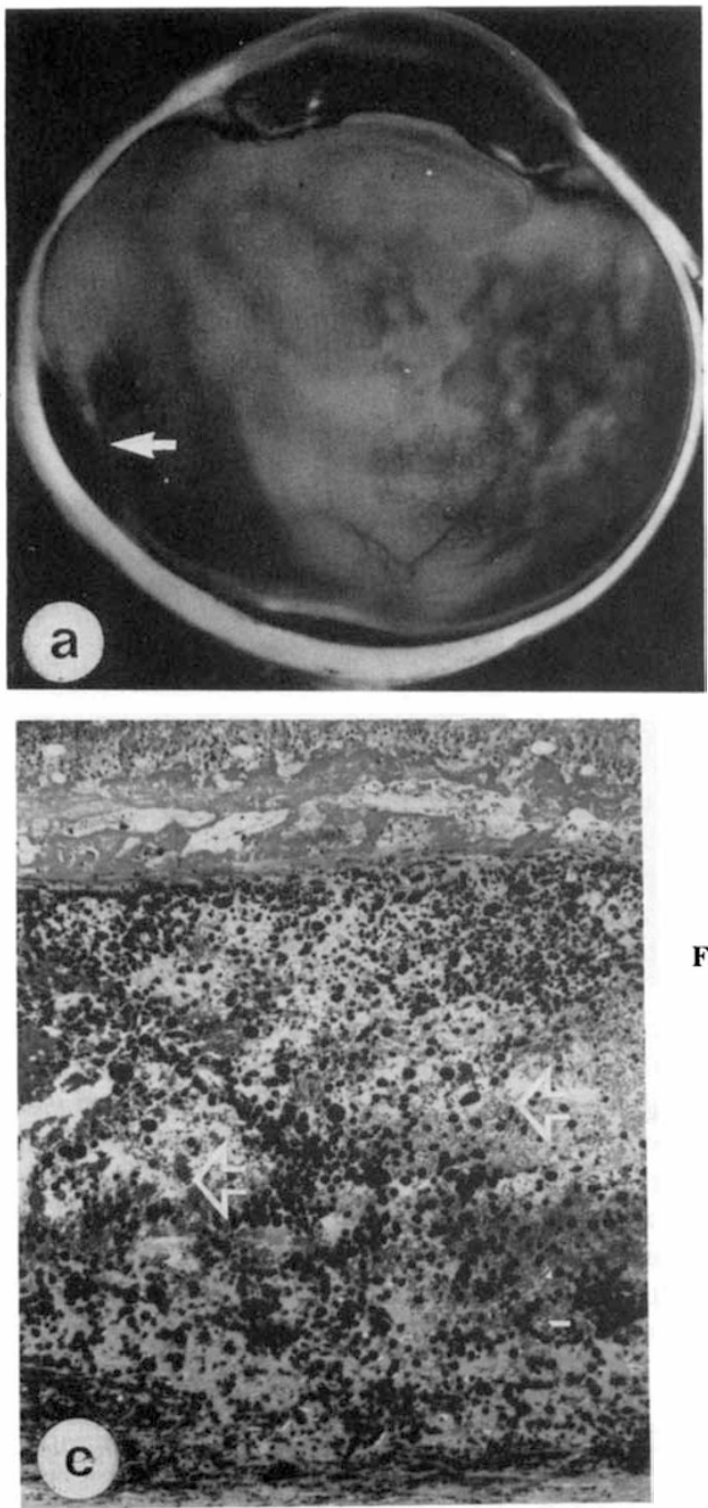

melanomacrophages (Fig. 2b) which were seen as large oval or spindle cells which contained numerous melanophagosomal bodies. Similar cells contained blood breakdown products in areas of haemorrhagic necrosis (Fig. 2c). Thus, the black mass shown in Fig. 2a was apparently composed of cells which were entirely of an inflammatory nature (Figs. 2b, c): the widespread necrosis was due in part to irradiation vasculopathy.

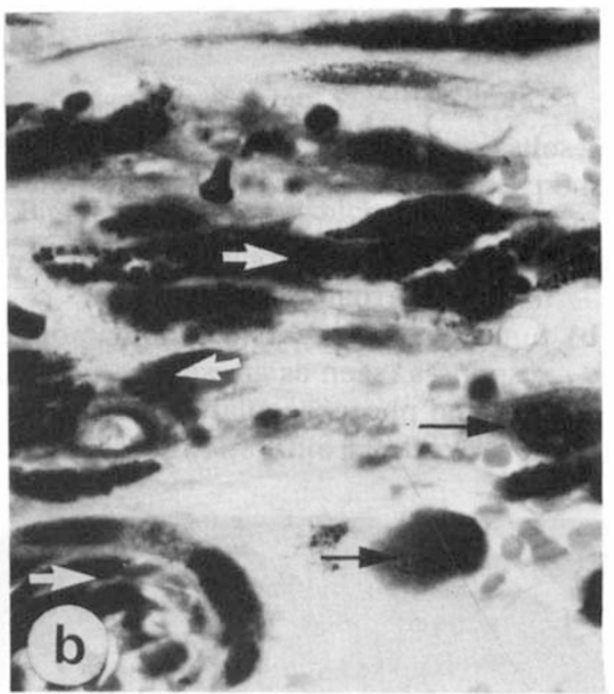

Fig. 2(a). A melanoma treated by an $R u^{106}$ plaque ( $\uparrow)$ followed by radiation retinopathy $(\times 3)$.

(b). The black choroidal mass contains large melanomacrophages located around blood vessels (white arrows): atypical cells are also present $(\uparrow)(H E \times 430)$.

(c). A low power shows the full thickness of the choroid with areas of haemorrhagic necrosis (open arrows) in addition to melanomacrophages beneath the necrotic retina $(H E \times 60)$. 
Convincing evidence of total extirpation of malignant melanoma cells was not obtained in this case or in others in which tumour necrosis was apparently achieved. Amongst the macrophages in such cases, there were isolated spindle cells containing fine melanin granules. These cells did not have the dendritic characteristics of normal choroidal melanocytes. Other cells (Fig. 2b) were large and oval and the nuclei showed irregular chromatin distribution and the nucleoli were large. The nature of these atypical cells is uncertain and the possibility that these cells were dormant tumour cells could not be excluded. A more convincing example of irradiation-induced 'dormancy' in tumour cells was seen in a melanoma which recurred at the posterior limit of an $\mathrm{Ru}^{106}$ plaque treatment area. In this

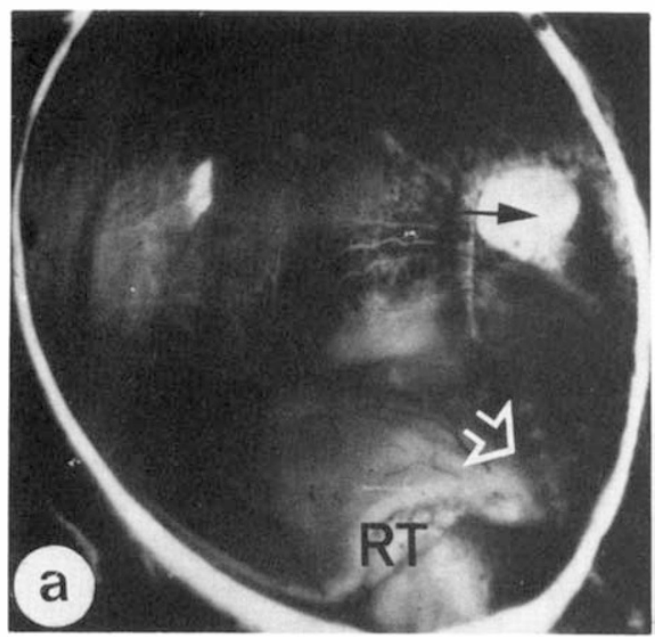

Fig. 3(a). The calotte from an eye which was initially biopsied ( $\uparrow$ ) and then treated with an $R u^{106}$ plaque at site shown (white arrow): recurrence of tumour growth $(R T)$ occurred posterior to the irradiated area $(\times 3)$.

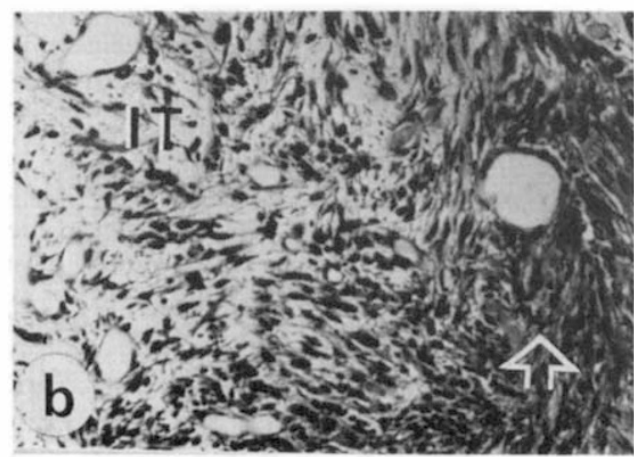

case (a 73 year old female patient in poor general health) a choroidal biopsy established the diagnosis of spindle $\mathrm{B}$ melanoma (Fig. 3a). In the enucleation specimen, there were two clear demarcation zones within the tumour. In the posterior untreated region where the tumour had continued to grow, the cells were closely packed spindle B melanoma cells. In the anterior irradiated part, the cells were heavily pigmented and extremely attenuated and there was a prominent collagenous extracellular space: mitotic figures were not found in this region (Figs. 3b, c).

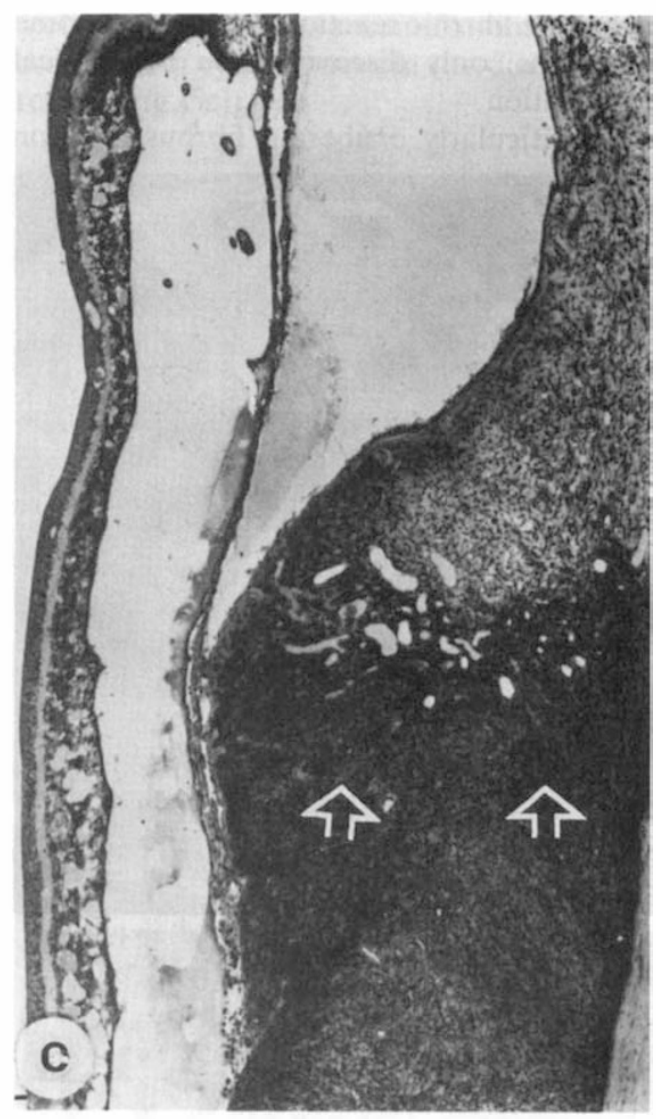

(b) and (c). Junction between the irradiated tumour (IT) and recurrent tumour to show the loose spacing of the dendritic melanocytes and melanomacrophages: compare with the densely packed typical spindle $B$ melanoma cells (white arrows) $(b \times 120 ; c \times 25$ : $H E)$. 
Round melanomacrophages were scattered amongst the spindle cells. The impression was that the loosely-spaced pigmented spindle cells represented a radioresistant component of the original tumour cell population.

\section{(c) Scleral fibrosis}

Large bands of white fibrous tissue grew beneath the surgical coloboma in many of the specimens examined. This fibrous tissue spread across the inner retina when there was retinal perforation. Amelanotic tumour was not easily recognisable macroscopically within the fibrotic reactions and was, in some specimens, only discovered on histological examination.

A particularly exuberant fibrous reaction was often seen after intensive photocoagulation therapy. In one case which was entirely treated by photocoagulation (Fig. 4a) fibrovascular tissue grew rapidly into the vitreous and had reached the lens by the time that the globe was enucleated. In addition to this complication, small pigmented nodules had persisted in the treated area. Histology of the pigmented tissue at the base of the fibrous band revealed coarsely pigmented melanomacrophages (Fig. 4b) and spindle and epithelioid cells which were widely spaced by collagenous tissue. The latter cells had the cytologic characteristics of malignant melanoma cells, i.e. fine melanin pigmentation and prominent nucleoli within irregular hyperchromatic nuclei (Fig. 4c).
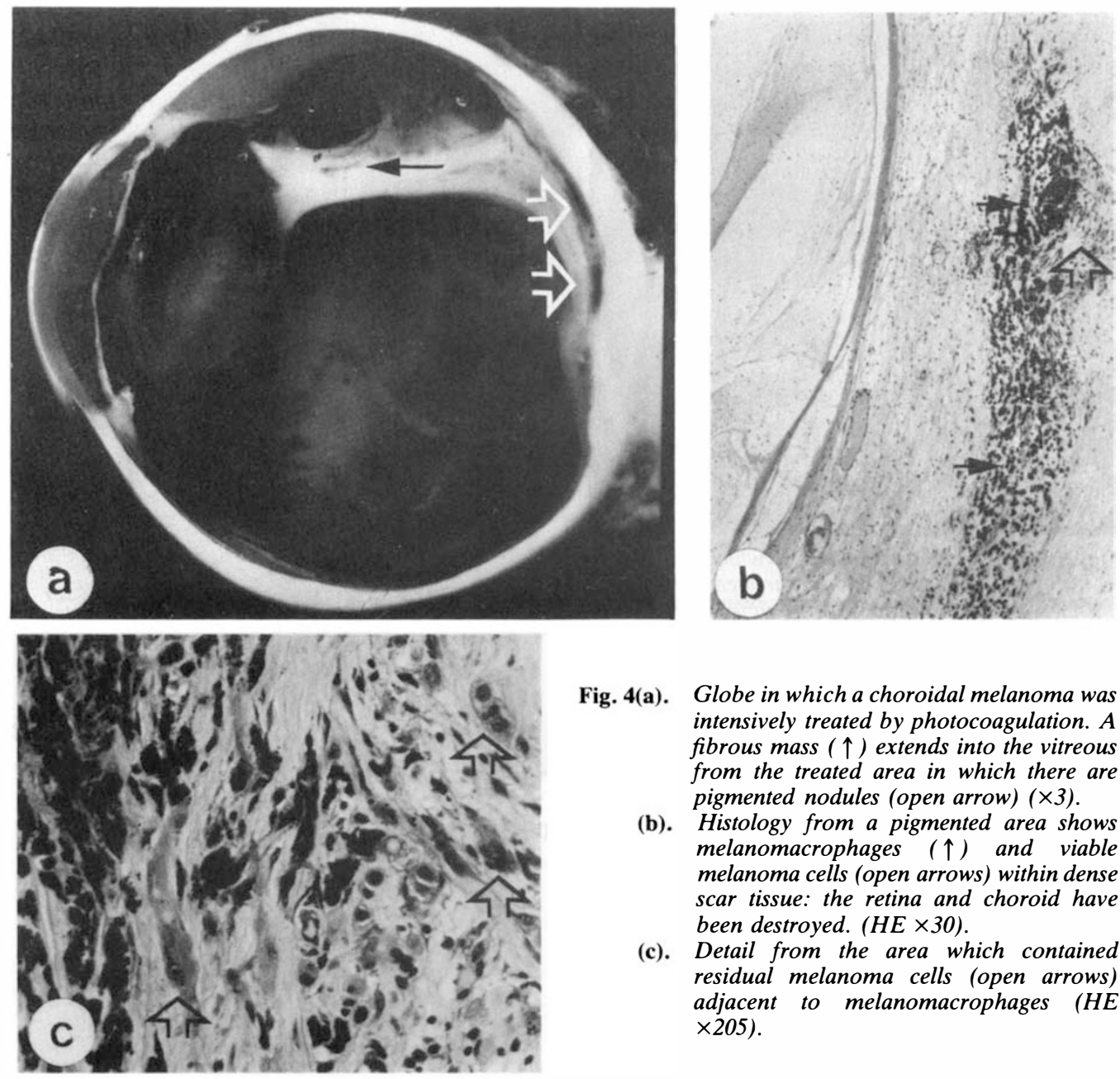

Fig. 4(a). Globe in which a choroidal melanoma was intensively treated by photocoagulation. A fibrous mass ( $\uparrow$ ) extends into the vitreous from the treated area in which there are pigmented nodules (open arrow) $(\times 3)$.

(b). Histology from a pigmented area shows melanomacrophages ( $\uparrow$ ) and viable melanoma cells (open arrows) within dense scar tissue: the retina and choroid have been destroyed. $(\mathrm{HE} \times 30)$.

(c). Detail from the area which contained residual melanoma cells (open arrows) adjacent to melanomacrophages (HE $\times 205)$. 


\section{(d) Reactionary changes in the RPE}

Simple hypertrophy and hyperpigmentation of the RPE was uncommon but this produced a diffuse darkening of the chorioretinal tissue which was disconcerting on macroscopic examination (Fig. 1a). The association with photoreceptor atrophy (Fig. 1c) suggests that the most likely cause was pre-existing retinal detachment followed by re-attachment. Hyperlipofuscinosis of the RPE (using UV microscopy) was not demonstrated.

Reactionary proliferation of the RPE was most commonly seen as a band of lightly pigmented tissue at the posterior limit of a surgical coloboma (Fig. 5a). Here the cuboidal and spindle cells (which retained the characteristic melanin granules of the RPE) were located within dense collagenous tissue (Fig. 5b). Such reactionary proliferation had the capacity to plug small retinal defects (Fig. 5b) and to fuse with the overlying gliotic retinal tissue.

Pigment speckling within the centre of a coloboma (Figs. 5a and c) was most commonly the result of proliferation of pigmented spindle cells which were often isolated within scar tissue. Heavier and coarser macroscopic pigment granules were the result of melanomacrophagic accumulations, in this case, within cystic spaces in the disorganised retina (Fig. 5c).
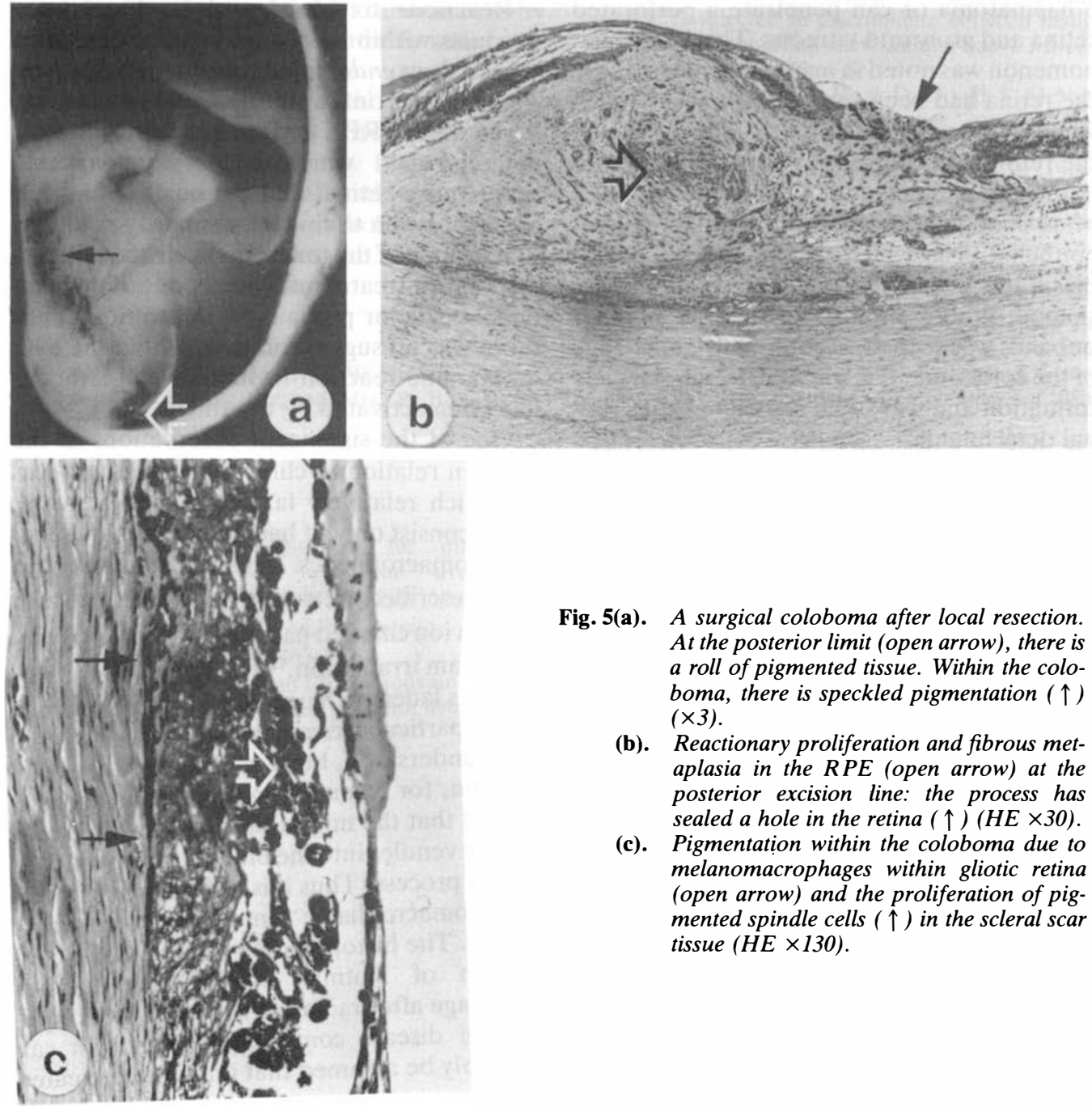

Fig. 5(a). A surgical coloboma after local resection. At the posterior limit (open arrow), there is a roll of pigmented tissue. Within the coloboma, there is speckled pigmentation ( $\uparrow$ ) $(\times 3)$.

(b). Reactionary proliferation and fibrous metaplasia in the RPE (open arrow) at the posterior excision line: the process has sealed a hole in the retina $(\uparrow)(H E \times 30)$.

(c). Pigmentation within the coloboma due to melanomacrophages within gliotic retina (open arrow) and the proliferation of pigmented spindle cells ( $\uparrow)$ in the scleral scar tissue $(H E \times 130)$. 


\section{Discussion}

The effects on the choroid and retina after a resection of a tumour and the adjacent choroid and sclera are to some extent predictable from information already available in the literature ${ }^{5,6}$ concerning the consequence of trauma. However, resection of a circular or oval area of choroid leaving a relatively undamaged retina would be unusual in the commonly encountered forms of mechanical trauma. It was of interest to see in the majority of cases, that the gliotic retina fused with the sclera and that the fusion inhibited fibroblastic proliferation. It is well recognised however that granulation tissue from the sclera and the edge of the torn choroid can grow into a haematoma or can penetrate a perforated retina and grow into vitreous. This latter phenomenon was noted in many specimens where the retina had been torn and it was also seen when photocoagulation had totally destroyed the retina and choroid. In terms of the problem of pseudomelanomas, fibrous scar tissue often contained proliferating retinal pigment epithelial cells which had undergone metaplasia and were lightly pigmented and spindleshaped. Proliferation to form visible nodules or bands was seen most commonly at the edge of the coloboma: this was akin to ringschwiele formation and was often associated with retinal detachment.

The origin of benign pigmented cells causing pigment speckling in the centre of a coloboma is somewhat puzzling and the best explanations are either that some choroidal melanocytes were left in the surgical bed postoperatively or that retinal pigment epithelium grew into the scar. It is however difficult to reconcile this suggestion with the generally accepted concept that the choroidal melanocyte is incapable of reactionary proliferation. However a recent study has shown that iris melanocytes can grow in the corneal stroma ${ }^{7}$ and it is possible that the proliferative capacity of choroidal melanocytes has been overlooked. Electron microscopy will be of value for the clarification of this point, since the melanosome content and cellular attachments of choroidal melanocytes differ from those of RPE cells. It is encouraging that there is now a potential for differentiating between the cells in benign and malignant melanocytic tumours of skin by means of immunohistochemistry using monoclonal antibodies $^{8,9}$. The application of this histological technology to reactionary versus tumour cell proliferation should prove rewarding in the future.

Many of the disturbances originating in the retinal pigment epithelium (hyperplasia and metaplasia) described in the present study have been documented previously ${ }^{10}$ as a response to many forms of insult. The observation that the monolayer contained excessive melanin granules, without hyperlipofuscinosis (Fig. 1c) is very unusual and the cause is obscure, although there is probably an association with photoreceptor atrophy.

Reactions to blood and its breakdown products within the eye have been described succinctly as endophthalmitis haemorrhagica. ${ }^{6}$ The cellular infiltrate was almost entirely derived from RPE and exogenous facultative macrophages, which have a remarkable capacity for retinal infiltration. It is of interest, in relation to this inflammatory reaction, that in none of the tumours which had failed to respond to treatment, was there a significant lymphocytic or plasma cell infiltration. Thus there was no suggestion that, within the eye, an immune reaction to tumour cell antigens had been activated by treatment.

One of the significant observations in the study in relation to clinical management was that such relatively large volumes of tissue could consist only of haemomacrophages and melanomacrophages. This phenomenon has been described previously as a consequence of helium ion charged particle therapy ${ }^{11}$ and proton beam irradiation. ${ }^{12}$ The behaviour of phagocytes laden with intra-cytoplasmic debris in these particular circumstances in vivo, is not fully understood, but it seems likely (by comparison, for example, with vitreous haemorrhage) that the migration of the swollen cells via the venules into the blood stream would be a slow process. Thus it is not surprising that a melanomacrophagic 'tumour' is slow to shrink in size. The histopathology supports the contention of Rotman etal. ${ }^{13}$ that 'tumour shrinkage after radiation is not a prime requisite for disease control'. Conversely it can probably be assumed that if a tumour treated by any means, increases in size, it is likely that 
the malignant cells have continued to proliferate.

One of the most difficult features in the histopathology of conservative management of melanomas has been the discrimination between reactionary fibroblastic activity or inflammatory mononuclear phagocyte infiltration and 'survival'-'dormancy' or 'reactivation' of tumour cells. Figures $2 b, 3 b$ and $4 c$ were included to illustrate some of the problems which have arisen in making this distinction and as yet, specific and valid criteria have not been established although immunohistochemistry offers some optimism. Manschot and van Striik ${ }^{14}$ have stressed (forcibly) that there are few acceptable histological reports of total extirpation of tumour cells by irradiation. This is understandable since successfully treated tumours (on clinical grounds), will be seen by pathologists in only exceptional circumstances. Even when specimens are made available by for example a road traffic accident, ${ }^{11}$ it would take a prodigious effort before the pathologist could be certain that there was not one viable tumour cell within the treated area.

It is a pleasure to thank Mr. J. A. W. Ralston, FIMLS for his technical expertise and Mrs. P. Bonnar for secretarial support.

\section{References}

${ }^{1}$ Ferry AP: Lesions mistaken for malignant melanoma in the posterior uvea. A clinicopathologic analysis of 100 cases with ophthalmoscopically visible lesions. Arch Ophthalmol 1964, 72: 463-9.

2 Shields JA and Zimmerman LE: Lesions simulating malignant melanoma of the posterior uvea. Arch Ophthalmol 1973, 89: 466-71.
${ }^{3}$ Zimmerman LE: Problems in the diagnosis of malignant melanomas of the choroid and ciliary body. Am J Ophthalmol 1973, 75: 917-29.

${ }^{4}$ Foulds WS, Damato BE, Burton RL: Local resection $v s$ enucleation in the management of choroidal melanoma. Eye (in press).

${ }^{5}$ Morris DA: Ocular trauma. In: Garner A, Klintworth GK eds. Pathobiology of Ocular Disease, New York, Dekker, 1982, pp 397-440.

${ }^{6}$ von Domarus D and Naumann GOH: Accidental and surgical trauma and wound healing of the eye. In: Naumann GOH, Apple DJ, eds. Pathology of the Eye, New York, Springer, 1986, pp 185-248.

${ }^{7}$ Ko MK, Um KB, Choe JK: Observations on iris melanocytes implanted in the cornea. Kor $J$ Ophthalmol 1987, 1: 23-5.

${ }^{8}$ Ruiter DJ, Dingjan GM, Steijlen PM, van BeverenHooyer M, de Graaf-Reitsma CB, Bergman W, van Muijen GNP, Warnaar SO: Monoclonal antibodies selected to discriminate between malignant melanoma and naevocellular naevi. $J$ Invest Dermatol 1985, 85: 4-8.

${ }^{9}$ Gown AM, Vogel AM, Hoak D, Gough F, McNutt MA: Monoclonal antibodies specific for melanocytic tumours distinguish subpopulations of melanocytes. Am J Path 1986, 123: 195-203.

${ }^{10}$ Morris DA and Henkind P: Pathological responses of the human retinal pigment epithelium. In: Zinn KM, Marmor eds. The Retinal Pigment Epithelium, Cambridge Mass, Harvard University Press, Cambridge Mass, 1979, pp 247-66.

${ }^{11}$ Grizzard WS, Torczynski E, Char DH: Helium ion charged particle therapy for choroidal melanoma. Histopathologic findings in a successfully treated patient. Arch Ophthalmol 1984, 102: $576-8$.

12 Ferry AP, Blair CJ, Gragoundas ES, Volk SC: Pathologic examination of a ciliary body melanoma treated with proton beam irradiation. Arch Ophthalmol 1985, 103: 1849-53.

${ }^{13}$ Rotman M, Long RS, Packer S, Moroson H, Galin MA, Char B: Radiation therapy of choroidal melanoma. Tr Ophthalmol Soc UK 1977, 97: 431-5.

${ }^{14}$ Manschot WA and van Striik R: Is irradiation a justifiable treatment of choroidal melanoma: An analysis of published results. $\mathrm{Br} \mathrm{J}$ Ophthalmol 1987, 71: 348-52. 